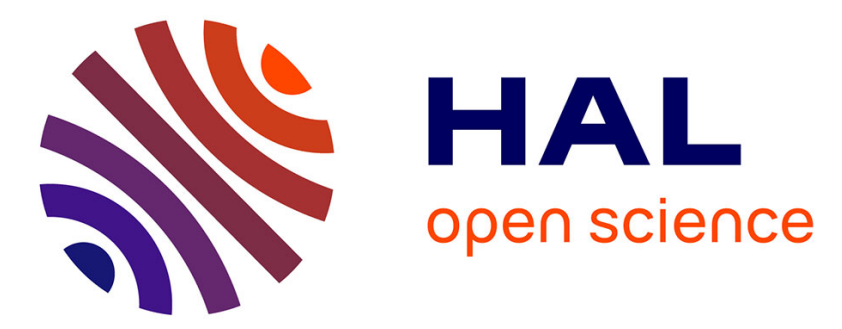

\title{
L'état d'urgence (1955-2005). De l'Algérie coloniale à la France contemporaine : destin d'une loi
}

Sylvie Thénault

\section{To cite this version:}

Sylvie Thénault. L'état d'urgence (1955-2005). De l'Algérie coloniale à la France contemporaine : destin d'une loi. Le Mouvement social, 2007, 218 (1), pp.63. 10.3917/lms.218.0063 . hal-02355811

\section{HAL Id: hal-02355811 \\ https://hal.science/hal-02355811}

Submitted on 12 Nov 2019

HAL is a multi-disciplinary open access archive for the deposit and dissemination of scientific research documents, whether they are published or not. The documents may come from teaching and research institutions in France or abroad, or from public or private research centers.
L'archive ouverte pluridisciplinaire HAL, est destinée au dépôt et à la diffusion de documents scientifiques de niveau recherche, publiés ou non, émanant des établissements d'enseignement et de recherche français ou étrangers, des laboratoires publics ou privés. 


\title{
L'ÉTAT D'URGENCE (1955-2005). DE L'ALGÉRIE COLONIALE À LA FRANCE CONTEMPORAINE : DESTIN D'UNE LOI
}

\author{
Sylvie Thénault
}

La Découverte | « Le Mouvement Social »

$2007 / 1 n^{0} 218$ | pages 63 à 78

ISSN 0027-2671

Article disponible en ligne à l'adresse :

https://www.cairn.info/revue-le-mouvement-social-2007-1-page-63.htm

Distribution électronique Cairn.info pour La Découverte.

(C) La Découverte. Tous droits réservés pour tous pays.

La reproduction ou représentation de cet article, notamment par photocopie, n'est autorisée que dans les limites des conditions générales d'utilisation du site ou, le cas échéant, des conditions générales de la licence souscrite par votre établissement. Toute autre reproduction ou représentation, en tout ou partie, sous quelque forme et de quelque manière que ce soit, est interdite sauf accord préalable et écrit de l'éditeur, en dehors des cas prévus par la législation en vigueur en France. Il est précisé que son stockage dans une base de données est également interdit. 


\section{L'état d'urgence (1955-2005). De l'Algérie coloniale à la France contemporaine : destin d'une loi}

\section{par Sylvie THÉNAULT*}

$\mathbf{L}$ e lundi 7 novembre 2005, Dominique de Villepin informait les Français de l'entrée en vigueur de l'état d'urgence. Toutefois, comme le président de la République dans son communiqué annonçant l'application de "la loi de 1955 ", il a évité d'employer "état d'urgence". "Utiliser ce terme lundi soir, c'était reconnaitre aux yeux du monde que la France était à feu et à sang ", confiait " un membre du gouvernement " (1). C'était, en effet, affirmer qu'un état d'exception était devenu indispensable, après une dizaine de jours de violences dans le pays. Le Premier ministre, prudent, ne parla que de "couvre-feu".

Mais qu'est-ce que l'état d'urgence? En un demi-siècle, les appréciations sur son compte n'ont pas varié. "Loi scélérate ", "loi de la terreur ", "état de siège fictif aggravé " pour les députés de gauche qui l'ont combattu, il y a cinquante ans (2); loi "stigmatisante, violente et inutile", synonyme de " guerre civile ", "complètement délirante de militarisation, de quadrillage outrancier, qui permet tous les dérapages ", renchérissent leurs héritiers d'aujourd'hui (3). De même, alors qu'en 1955, le gouvernement la justifiait par la situation en Algérie où, affirmait-il, "la prolongation de l'insécurité trouve son origine essentielle dans l'insuffisance des moyens de droit qui n'ont pas été conçus pour des périodes insurrectionnelles" (4), le gouvernement actuel explique qu' i il est important pour l'État d'avoir les moyens de recourir à tous les outils nécessaires pour restaurer très vite la paix dans les quartiers " (5).

* Historienne, chargée de recherche au CNRS (Centre d'Histoire Sociale du XXe siècle).

(1) Cité par Le Monde, le 9 novembre 2005.

(2) Cf. notamment les interventions de Raymond Guyot (communiste), Alice Sportisse (communiste) et Francis Vals (socialiste) à l'Assemblée nationale, les 30 et 31 mars 1955.

(3) Déclarations respectives du Parti communiste français (PCF), des Verts et de la Ligue communiste révolutionnaire (LCR). Le Monde, 10 et 14 novembre 2005.

(4) D'après l'exposé des motifs du projet de loi.

(5) Déclaration de "l'entourage du Premier ministre", citée par Le Monde, le 9 novembre 2005.

Le Mouvement Social, n 218, janvier-mars 2007, @ Les Éditions de l'Atelier/Éditions Ouvrières 
Il est évident que, pour divergentes qu'elles soient, de telles déclarations renvoient à une même réalité : celle d'un texte instaurant un régime d'exception. L'état d'urgence permet au représentant de l'État - préfets dans les départements et, en 1955, gouverneur général en Algérie - d'instaurer un couvre-feu, de réglementer la circulation et le séjour dans certaines zones géographiques, de prononcer des interdictions de séjour et des assignations à résidence contre des individus. Il autorise aussi la fermeture de lieux publics, tels que des salles de spectacle, des cafés ou des salles de réunion, l'interdiction de réunions ou rassemblements, la confiscation des armes détenues par des particuliers, le contrôle de la presse, des publications, des émissions de radio ou encore des projections de cinéma et des représentations théâtrales. Enfin, il dessaisit la justice de prérogatives essentielles : les autorités administratives obtiennent le droit de pratiquer des perquisitions, de jour comme de nuit, et la justice militaire peut être déclarée compétente.

En 1955, de telles mesures répondaient spécifiquement aux besoins des autorités confrontées au développement d'une insurrection en Algérie. Comment, alors, cette loi, votée comme une loi de circonstance, peut-elle être remise en vigueur, un demi-siècle plus tard, dans une conjoncture totalement différente ? Le recours à l'état d'urgence serait-il l'indice d'un héritage colonial non assumé mais resurgissant à la faveur de l'actualité ? Ce serait négliger les usages et transformations du texte entre 1955 et 2005, qui l'inscrivent dans l'histoire de la répression en régime républicain.

\section{5, une loi pour l'Algérie}

Au moment du vote de la loi, l'insurrection algérienne, qui compte cinq mois d'existence, se manifeste par l'installation de maquis, en particulier dans la région des Aurès, où la France a dû engager des milliers d'hommes dans de véritables opérations militaires. Elle se manifeste aussi par la multiplication d'attentats, scrupuleusement suivis par le ministère de l'Intérieur qui recense chaque mois plusieurs dizaines de sabotages de lignes et voies de communication, d'incendies criminels, d'attaques à main armée, d'explosions de bombes, de destructions de marchandises, de matériel, de bétail, etc. (6).

Le Front de libération nationale (FLN), par ailleurs, créé par un noyau d'activistes décidés à se lancer dans la lutte armée, se présente comme une "constellation d'appareils fonctionnels décentralisés, agissant sans référence à une stratégie commune " (7). Il s'attelle à une double tâche de recrutement de militants et de persuasion de la population. Entré en action sans structure militante et sans cadres, en effet, le FLN n'est pas un parti ; il est entièrement à construire par le ralliement d'hommes venus principalement, dans un premier temps, du mouvement nationaliste alors existant: le Mouvement pour le triomphe des libertés démocratiques

(6) Relevés mensuels, Archives nationales (AN), F1A 4811 (sous dérogation).

(7) M. HARBI, Le FLN, miroir et réalité, Paris, Jeune Afrique, 1980, p. 172. 
(MTLD). La survie d'une insurrection déclenchée sans un travail préalable de ralliement des masses, en outre, passe par la recherche du soutien des Algériens, appelés à s'engager au service d'une organisation parfaitement inconnue d'eux.

"Insuffisance des moyens de droit " dit l'exposé des motifs de la loi d'état d'urgence. Effectivement, les autorités françaises sont face à un véritable défi, car la réponse militaire, seule, ne suffit pas. Il leur faut aussi démanteler tout réseau formé pour commettre des attentats ou apporter au FLN un soutien quelconque, contrarier la constitution d'une organisation politique, entraver celle d'une véritable armée, décourager l'adhésion des Algériens à cette cause, punir leur participation à de telles activités. C'est un travail de police - perquisitions, arrestations, interrogatoires, recherche de renseignements, contrôle des déplacements et agissements des individus considérés comme suspects... - qu'il faut mener; ce sont des opinions qu'il faut combattre, une propagande qu'il faut empêcher; ce sont des hommes et des femmes qu'il faut dissuader de passer à l'action, par tout un arsenal de mesures préventives à l'égard de ceux qui seraient tentés de le faire, et par la répression exemplaire de ceux qui ont franchi le pas de l'engagement. L'état d'urgence, avec son cortège de mesures permettant de contrôler l'espace, les idées, les individus, est alors créé pour répondre à la spécificité de cette situation. Il se présente comme affranchi du respect des libertés individuelles et collectives, perçues comme autant de contraintes sources d'inefficacité face à l'ennemi. Il dote les autorités de larges pouvoirs tout en leur évitant de recourir à l'état de siège, seule alternative existant au droit commun du temps de paix.

En effet, la proclamation de l'état de siège, transférant tous les pouvoirs à l'armée, aurait posé de multiples problèmes. Sur un plan pratique, d'abord, le dessaisissement total des autorités civiles n'était pas souhaité par les deux gouvernements concepteurs de l'état d'urgence, celui de Pierre Mendès France, jusqu'en février 1955, puis celui d'Edgar Faure (8). C'est que tous deux pensaient indispensable de réduire les inégalités marquant l'Algérie pour mieux combattre le nationalisme. Jacques Soustelle, le gouverneur général nommé à Alger par Pierre Mendès France et confirmé par son successeur, reçut pour mission d'y consacrer ses réflexions. Dans la continuité d'un réformisme colonial très ancré dans la gauche française, ces hommes analysaient la misère des Algériens, leur analphabétisme, leur privation des droits politiques... comme autant de maux faisant le jeu des indépendantistes et condamnant, à terme, la survie de l'Algérie française. A leurs yeux, la lutte contre l'insurrection ne passait pas que par la répression de ses acteurs, mais aussi par une politique de développement du pays. Or l'élaboration et la mise en œuure de réformes nécessitaient le maintien d'autorités administratives civiles en état d'agir. Les gouvernements ont refusé l'état de siège pour éviter de les désorganiser.

Un tel choix reposait par ailleurs sur l'idée que le FLN pouvait être assez vite vaincu, que l'Algérie française survivrait et que le temps des réformes arriverait dans

(8) Lors des débats à l'Assemblée nationale, le 31 mars 1955, les ministres de l'Intérieur de ces deux gouvernements, François Mitterrand et Maurice Bourgès-Maunoury, reconnaissent leur participation à l'élaboration de l'état d'urgence. 
des délais relativement courts. Cette perspective, erronée mais compréhensible à un moment où les nationalistes viennent seulement de lancer leur combat, explique aussi la mise au point de l'état d'urgence. Les gouvernements misaient sur un étouffement rapide de l'insurrection, présentée comme un "désordre" provoqué par "quelques bandes organisées de hors-la-loi, numériquement peu importantes " et concentrées " dans des zones dont la structure naturelle est particulièrement propice à des actions de guérilla " (9). Il est vrai qu'il a fallu attendre le soulèvement du Nord-Constantinois, à partir du 20 août 1955, pour que le basculement dans la guerre apparaisse nettement comme irréversible. Dans ces conditions, pourquoi recourir, dès le début de l'année 1955, à l'état de siège?

Politiquement, enfin, la proclamation de l'état de siège était inopportune. Elle aurait entrainé une qualification militaire des nationalistes en action, alors qu'il importait de les présenter comme des délinquants et des criminels passibles des tribunaux, pour leur enlever toute légitimité. "Les hommes qui commettent ces attentats contre les personnes et les biens ne sauraient en aucun cas être considérés comme ayant un caractère militaire ", estimait ainsi le ministre de l'Intérieur de Pierre Mendès France, François Mitterrand, une dizaine de jours après la Toussaint 1954, au diapason de tous les hommes politiques de l'époque (10). Mais surtout la reconnaissance d'un état de guerre aurait suggéré que l'Algérie, siège d'un conflit armé avec la France, était un espace détaché du reste du pays alors que, constituée de départements à l'identique de la métropole, elle y était soudée. L'exposé des motifs de la loi d'état d'urgence affirme ainsi clairement que "l'Algérie, partie intégrante du territoire national, ne peut se voir dotée d'un régime d'exception".

C'est là, alors, que le bât blesse : comment soumettre l'Algérie à l'état d'urgence sans s'exposer à l'accusation d'un traitement discriminatoire de ce territoire, sans toucher au dogme de son rattachement à l'espace français? Les gouvernements ont trouvé une parade, directement à l'origine de l'inscription définitive de cet état dans le droit français. Dans un premier temps, en effet, le ministère de l'Intérieur a rassemblé des propositions provenant de diverses sources, interrogées sur les mesures à prendre pour le rétablissement de l'ordre : gouvernement général de l'Algérie, préfectures, ministère de la Justice (11). L'idée de certaines mesures remonterait ainsi aux lendemains des deux tremblements de terre qui ont touché la région d'Orléansville en septembre 1954, cette catastrophe ayant été suivie de pillages qui auraient inquiété les autorités et les auraient alertées sur leur dénuement face à un tel désordre (12). Puis, une fois les suggestions retenues synthétisées dans un projet de loi, le gouvernement d'Edgar Faure a présenté le texte aux députés, sous l'intitulé d'" état d'urgence". L'opposition, menée essentiellement par les communistes et les

(9) Exposé des motifs de la loi d'état d'urgence.

(10) Lettre à Jean-Michel Guérin de Beaumont, ministre de la Justice, 13 novembre 1954, AN, BB18 4226 (sous dérogation).

(11) D'après une note sur l'état d'urgence du 28 février 1955. AN, F1A 4806 (sous dérogation).

(12) D'après les déclarations du ministre de l'Intérieur, Maurice Bourgès-Maunoury, à l'Assemblée nationale, le 31 mars 1955. 
socialistes, ne suffisant pas à en bloquer l'adoption, ce texte, remodelé par quelques amendements parlementaires, est devenu la loi no 55-385 du 3 avril 1955 (13).

Or, selon son article $1^{\text {er }}$, "l'état d'urgence peut être déclaré sur tout ou partie du territoire métropolitain, de l'Algérie ou des départements d'outre-mer, soit en cas de péril imminent résultant de graves atteintes à l'ordre public, soit en cas d'événements présentant, par leur nature et leur gravité, un caractère de calamité publique ". Ainsi, officiellement, il ne vise pas l'insurrection algérienne. Il est un nouvel état d'exception créé de toutes pièces, introduit dans le droit français par cette loi, mais applicable à n'importe quelle portion du territoire national. Il a été instauré en Algérie dans un second temps, conformément aux prescriptions de l'article 15. Celui-ci, déclarant l'état d'urgence "sur le territoire de l'Algérie pour une durée de six mois", prévoyait un décret pour fixer "les zones dans lesquelles [il] recevra application". Choisissant d'adapter au mieux sa réplique, en tentant de circonscrire les régions gagnées par la guerre, le gouvernement d'Edgar Faure procéda alors en trois étapes: un premier décret, le 6 avril, s'en tenait à certaines zones du Constantinois, avant qu'un deuxième, le 19 mai, couvre tout l'Est du pays, ainsi que la région de Tlemcen, près de la frontière marocaine. Ce n'est que le 28 août 1955, après le soulèvement marquant un point de non-retour dans l'engrenage du conflit, qu'un troisième décret appliqua l'état d'urgence dans toute l'Algérie.

Il n'y fut en vigueur, cependant, que trois mois. Il était en effet prévu que l'état d'urgence cesserait en cas de dissolution de l'Assemblée nationale, ce que décida Edgar Faure, le 30 novembre 1955. Cette courte durée ne l'empêcha pas de produire tous ses effets, les autorités utilisant largement les possibilités du texte : couvrefeu, saisies de journaux, perquisitions, interdictions de séjour et internements par milliers, jugements rendus par les tribunaux militaires de même... Après la fin de son application en Algérie, pourtant, la loi n'aurait-elle pas pu tomber en désuétude? Deux ans et demi plus tard, au contraire, les événements du 13 mai 1958 allaient provoquer sa déclaration en métropole.

\section{8-1963, une loi pour un pouvoir menacé}

L'occupation du gouvernement général à Alger, la substitution d'un Comité de salut public rassemblant civils et militaires aux autorités légales, l'appel au général de Gaulle et sa réponse favorable suscitent l'affolement à Paris. Les factieux se préparent-ils à prendre le pouvoir dans la capitale ? Quelle confiance accorder au Général ? Le 16 mai, Pierre Pflimlin, qui a été investi à la hâte par l'Assemblée, demande aux députés de déclarer l'état d'urgence en métropole. Cette fois, les députés communistes et socialistes soutiennent sa demande.

(13) L'état d'urgence est adopté par 361 voix contre 227. Les voix défavorables émanent des communistes et des socialistes, rejoints par des députés du Parti radical, du Mouvement républicain populaire (MRP) et de l'Union démocratique et socialiste de la résistance (UDSR). 
En 1955, pourtant, la possibilité de déclarer l'état d'urgence en métropole avait été au cœur de l'argumentation du Parti communiste contre le texte. Alors que les socialistes avaient fondé leur opposition sur la seule situation en Algérie, en expliquant que celle-ci ne requérait pas des mesures aussi graves, les communistes avaient affirmé que le gouvernement ne faisait que profiter de la conjoncture algérienne pour introduire dans le droit français un nouvel état d'exception, utilisable dans d'autres circonstances, avec l'intention, précisément, d'y recourir contre le mouvement ouvrier. Pour Alice Sportisse, par exemple, née en Algérie et député d'Oran depuis dix ans, l'insurrection lancée par le FLN n'était que "le prétexte choisi pour permettre au gouvernement de se forger une arme redoutable contre le peuple de France luimême" (14). "Il ne faut pas, mesdames, messieurs, que l'arbre cache la forêt et que certains d'entre vous, croyant voter un texte destiné à une colonie - encore que son contenu soit d'une gravité lourde de conséquences -, puissent se rendre complices d'une entreprise gouvernementale dirigée vers un autre but ", insistait-elle. L'argument était logique, à partir du moment où elle avait affirmé que le gouvernement "n'avait nullement besoin de faire voter de tels textes" pour faire face aux événements en Algérie. Il se comprend, aussi, dans un parti qui, de sa création à 1953, fut accusé à cinq reprises de complot contre la sûreté de l'État et dont la plupart des dirigeants furent poursuivis en justice, voire emprisonnés (15).

En des termes différents mais en accord sur le fond, André Cheneboit, ancien magistrat et avocat, rédacteur en chef du Monde depuis sa création, avait aussi alerté l'opinion sur "le caractère extraordinaire " de l'état d'urgence. Se demandant "si l'on ne braque pas un canon pour écraser une mouche ", il enfonçait le clou après avoir énuméré les dispositions du projet de loi : "Voilà, on l'avouera, qui est bien gros s'il ne s'agit que de réprimer les coups de main des fellagas (sic) de l'Aurès ". Et il concluait de façon prémonitoire : "On réfléchira en tout cas sur l'usage que pourraient faire de pareils textes un gouvernement et une Assemblée dans une situation actuellement hors de prévision" (16).

Ce n'est donc pas le moindre des paradoxes que de voir les forces de gauche voter l'article unique proposé par le gouvernement de Pierre Pflimlin en mai 1958 : l'état d'urgence " est déclaré sur l'ensemble du territoire métropolitain pour une durée de trois mois à compter de la promulgation de la présente loi " (17). Communistes et socialistes représentent en effet 236 voix sur un total de 462 votes favorables au texte - soit la moitié - et leurs groupes ont fait preuve d'une grande discipline, une voix seule faisant défaut (18). C'est que, à leurs yeux, la menace pesant sur le régime républicain cautionne mieux que tout autre motif le recours à un état d'exception. Marcel-Edmond Naegelen, l'ancien gouverneur général de l'Algérie connu pour le

(14) A l'Assemblée nationale, le 31 mars 1955.

(15) F. MONIER, "L'État face à la contestation communiste", in M.O. BARUCH et V. DuCLERT (dir.), Serviteurs de l'État, une histoire politique de l'administration française, 1875-1945, Paris, La Découverte, 2000, p. 427-438.

(16) Tribune en première page du Monde, le 24 mars 1955.

(17) Texte présenté à l'Assemblée nationale le 16 mai 1958.

(18) Le texte a trouvé l'assentiment général des forces de gauche et du centre, avec, outre les communistes et les socialistes, la quasi-totalité des progressistes, des radicaux, des élus de l'UDSR et du MRP. 
truquage des élections de 1948 sous son autorité, que le groupe socialiste a mandaté pour expliquer sa position, affirme ainsi à la tribune "très calmement, mais très résolument, sa volonté de défendre contre toute entreprise l'unité nationale et les institutions républicaines" (19). Quant à Jacques Duclos, pour le Parti communiste, il cite Saint-Just: "Pas de liberté pour les ennemis de la liberté". Présentant "la classe ouvrière" et "les forces populaires" comme "le suprême recours pour la défense de la République ", il demande une utilisation ciblée de l'état d'urgence, contre les seuls "factieux". Au contraire, "chacun de nous peut bien comprendre qu'il ne serait en aucun cas possible, face aux dangers fascistes se présentant devant le peuple de notre pays, d'empêcher les travailleurs et les républicains de se dresser vigoureusement contre les tenants de la dictature mettant la République en danger " (20).

Cette fois encore, l'état d'urgence n'est déclaré que pour une courte durée. La commission de l'Intérieur de l'Assemblée nationale avait ajouté à l'article proposé par le gouvernement de Pierre Pflimlin un alinéa prévoyant que les pouvoirs résultant de l'état d'urgence "seraient caducs en cas de changement de gouvernement " et ce pour garder un contrôle sur leur utilisation. C'était obliger toute nouvelle équipe ministérielle à consulter l'Assemblée et à obtenir son assentiment avant de disposer de cet état d'exception. Or le général de Gaulle, finalement intronisé le $1^{\text {er juin } 1958,}$ ne demanda pas la reconduction de l'état d'urgence, qui fut donc en vigueur moins de deux semaines et dont la portée resta minime.

Le nouveau chef de l'État, devenu le premier président de la Ve République, n'hésite pas, un peu plus tard, à réformer la loi de 1955, à un moment où, pourtant, il n'est absolument pas question d'y recourir. Ainsi, le 15 avril 1960, une ordonnance modifie la procédure de déclaration de l'état d'urgence (21). Désormais le gouvernement peut en décider seul, par décret, pour une durée de douze jours. Au-delà, l'Assemblée retrouve ses prérogatives puisqu'il lui revient de dire si l'état d'urgence doit être prorogé et pour quelle durée. La comparaison avec l'état de siège, véritable leitmotiv de la dénonciation de l'état d'urgence, devient plus que jamais d'actualité. C'est que les conditions de sa proclamation, définies par l'article 36 de la Constitution de la V'République, sont strictement identiques : "L'état de siège est décrété en conseil des ministres. Sa prorogation au-delà de douze jours ne peut être admise que par le Parlement".

L'écart entre ces deux textes est alors réduit. L'état de siège, défini par la loi du 9 août 1849, permet à l'autorité militaire de disposer des pouvoirs de police, de perquisitionner de jour et de nuit, d'éloigner toute personne de son domicile, de chercher et confisquer armes et munitions, d'interdire les publications et réunions. Il rend également la justice militaire compétente. Reste une différence fondamentale, néanmoins, qui explique la modification de l'état d'urgence intervenue en avril 1960 :

(19) Assemblée nationale, séance du 16 mai 1958.

(20) Assemblée nationale, séance du 16 mai 1958.

(21) Ordonnance n 60-372 du 15 avril 1960, fondée sur la loi du 4 février 1960 qui autorise le gouvernement à prendre, par ordonnance, toutes mesures "nécessaires pour assurer le maintien de l'ordre, la sauvegarde de l'Etat et de la Constitution, la pacification et l'administration de l'Algérie". 
au contraire de l'état de siège, l'état d'urgence n'accorde aucun pouvoir à l'armée. Ce sont les autorités civiles qui disposent des mesures exceptionnelles qu'il autorise. Or l'ordonnance du 15 avril 1960 intervient dans un contexte où la confiance du pouvoir en son armée s'est érodée, au point qu'une opération d'élimination des cadres et organismes militaires suspects de manquer de loyauté est en cours.

Il faut donc revenir à l'événement majeur du début de cette année 1960, en janvier, pour comprendre la modification de la loi de 1955 : la semaine d'émeutes dites des "Barricades ", à Alger, pendant laquelle les ultras de l'Algérie française ont tenu la rue, bouclant tout un quartier du centre-ville, pour protester contre l'autodétermination qu'a décidée le général de Gaulle. Or ils ont bénéficié de la complicité de hauts responsables militaires dans l'organisation de leur révolte, ainsi que de la complaisance des soldats chargés de les encercler mais qui, fraternisant avec eux, laissaient hommes et vivres circuler. Après la reddition des leaders des insurgés, sanctions et réorganisation ont visé l'armée.

La modification de la loi de 1955 par l'ordonnance du 15 avril 1960 prouve que le général de Gaulle, chef de l'État devenu la cible de tous ceux qui ne peuvent se résoudre à la perte de l'Algérie, a tiré un constat lucide de ces événements : il ne peut plus compter sur le loyalisme des militaires. Il faut qu'en cas de nouveau soulèvement contre le pouvoir, les autorités civiles elles-mêmes puissent disposer, facilement et rapidement, de moyens exceptionnels. Or, si l'état d'urgence leur fournit un arsenal efficace, les modalités de sa déclaration, prévoyant un vote du Parlement, deviennent trop lourdes; d'où leur réforme et la totale liberté accordée au gouvernement pour agir.

L'état d'urgence équivaut alors à un état de siège contournant l'armée, soit un état d'exception permettant de faire face à tout péril sans recourir à des militaires qui ont perdu la confiance de l'autorité politique. Au contraire, le général de Gaulle s'appuie sur les autorités civiles. En Algérie, ainsi, en cette année 1960, il retire aux militaires les pouvoirs gagnés à la faveur du changement de régime : après avoir réintroduit les IGAMES, il confie la direction de la police à un préfet, Jacques Aubert, et rend aux préfets et sous-préfets toutes leurs attributions (22). En métropole, il a fait le choix, en 1958, de reconduire les responsables policiers et les préfets en place, s'assurant le contrôle de ces derniers par les nominations et mutations ultérieures (23). Il a aussi maintenu le préfet de police de Paris, Maurice Papon, appelé à cette fonction après la fronde policière du 13 mars 1958, pour reprendre en main un corps de police dont le loyalisme donnait des signes de faiblesse. En métropole, dans les milieux policiers comme au sein du corps préfectoral, la perte de confiance dans le régime précédent profite au général de Gaulle, qui incarne la restauration de l'autorité du pouvoir politique après des années d'usure.

(22) M. HARDy, H. Lemoine et T. Sarmant, Pouvoir politique et autorité militaire en Algérie française. Hommes, textes, institutions, 1945-1962, Paris, SHAT/L'Harmattan, 2002.

(23) Sur la police : J.-M. BERLĖEE, " Un maintien de l'ordre entre naufrage des principes démocratiques et faillite de l'Etat?", in S. BeRSTeIn, P. Milza et J.-F. SIRINELLI (dir.), Michel Debré, Premier ministre, 1959-1962, Paris, PUF, 2005, p. 543. Sur les préfets : J. AUBERT, "L'administration du territoire", in INSTITUT CHARLES DE GAULle (dir.), De Gaulle et le service de l'État, Paris, Plon, 1977, p. 281 et suiv. 
L'ordonnance du 15 avril 1960 témoigne d'une anticipation remarquable sur les événements du printemps suivant, lorsque, au matin du 22 avril 1961, Alger se réveille aux mains des militaires. Le jour même, à Paris, le conseil des ministres décrète l'état d'urgence en métropole pour se donner les moyens de contrecarrer toute extension de la tentative de putsch hors du sol de l'Algérie. L'état d'urgence allait alors connaitre sa durée d'application la plus longue et la moins contrôlée, puisqu'il est resté en vigueur jusqu'au 31 mai 1963, soit deux ans durant et au-delà de la fin de la guerre en Algérie, où le cessez-le-feu a été proclamé le 19 mars 1962 et l'indépendance reconnue le 3 juillet suivant; sa plus longue durée d'application donc, et ce en dehors de toute intervention parlementaire.

Suivant les prescriptions de l'ordonnance du 15 avril 1960, en effet, la déclaration de l'état d'urgence par le conseil des ministres, le 22 avril 1961, aurait dû être confirmée par l'Assemblée nationale. Mais c'était compter sans l'entrée en vigueur de l'article 16 de la Constitution, à la faveur du putsch. Cet article accordant au président de la République tous les pouvoirs, le général de Gaulle signa le 24 avril 1961 une série de "décisions " publiées au Journal Officiel, dont celle-ci : " La durée de l'état d'urgence, déclaré et mis en application par les décrets susvisés du 22 avril 1961, est prolongée jusqu'à nouvelle décision ". Puis, le 29 septembre 1961, juste avant la fin de l'application de l'article 16, une ultime "décision " prolongea l'état d'urgence jusqu'au 15 juillet 1962. A cette date, cependant, l'indépendance de l'Algérie ayant été reconnue, le dernier carré des irréductibles de l'Algérie française, au sein de l'Organisation armée secrète (OAS), menaçait désormais directement la personne même du chef de l'État. Bénéficiant alors, une fois de plus, d'une loi lui accordant toute facilité pour appliquer ses résolutions en matière de politique algérienne, ce dernier prorogea de nouveau l'état d'urgence, jusqu'au 31 mai 1963 (24).

Ce long épisode métropolitain interroge doublement: quelle a été, alors, la portée de l'état d'urgence? et surtout, une fois déclaré et trois fois renouvelé au titre d'une conjoncture restée menaçante pour le pouvoir légal, quand et comment pouvait-il prendre fin? En 1962 et 1963, ainsi, la presse signale régulièrement que le pays vit toujours sous le régime de l'état d'urgence, comme si les effets concrets de ce dernier restaient suffisamment limités ou invisibles pour que les Français l'oublient (25). En même temps, ces rappels récurrents posaient implicitement la question de savoir quand il cesserait.

La portée du texte est très difficile à mesurer, en raison de la multiplicité des mesures d'exception utilisées par le général de Gaulle pour réprimer les opposants à sa politique algérienne et sauvegarder le régime de la Ve République. Le chef de

(24) Ordonnance nº2-797 du 13 juillet 1962, fondée sur la loi du 13 avril 1962, approuvée par référendum. Cette loi permet au gouvernement de prendre toute mesure en vue d'assurer l'application des déclarations du 19 mars 1962, c'est-à-dire les Accords d'Évian, et en particulier le cessez-le-feu en Algérie.

(25) A titre d'exemple, Le Monde rappelle, le 18 janvier 1962, que l'état d'urgence est toujours en vigueur ; il s'interroge, dans son édition du $1^{\text {er }}-2$ juillet 1962 , sur le maintien éventuel de l'état d'exception ; il signale, le 22 décembre 1962, la saisie d'un livre en vertu de ces dispositions; il titre son numéro du 6-7 janvier 1963 : "Deux ans sous l'état d'urgence". Et le 10 mai 1963, enfin : "L'état d'urgence, qui prend fin le 31 mai, va-t-il être de nouveau prorogé ?". 
l'État, ainsi, dispose des pouvoirs spéciaux qui, votés à la demande de Guy Mollet pour l'Algérie le 16 mars 1956, ont été étendus à la métropole le 26 juillet 1957 et reconduits par l'Assemblée en faveur de tous les gouvernements postérieurs, dont celui du général de Gaulle le 2 juin 1958 (26). L'article 16 lui-même ne lui a pas seulement permis de prolonger l'état d'urgence sans consulter les députés. Il lui a aussi servi, par exemple, à porter la durée de la garde à vue à quinze jours et à étendre la pratique de l'internement administratif aux partisans de l'Algérie française, deux "décisions" signées le 24 auril 1961 et confirmées, comme l'état d'urgence, en septembre 1961 et en juillet 1962. Evaluer les effets concrets du seul état d'urgence, sur le territoire métropolitain, entre 1961 et 1963, supposerait donc, au préalable, de démêler les mesures découlant de cet état d'exception et les autres.

La question de savoir quand l'état d'urgence doit cesser, par ailleurs, émerge de façon polémique en janvier 1963. A l'occasion du vote du budget de la Justice, un député MRP, professeur de droit, Paul Coste-Floret, développe à la tribune de l'Assemblée la thèse suivant laquelle l'état d'urgence, en fait, a déjà pris fin. Cet ancien résistant, ayant joué un rôle au sommet dans les institutions de la France Libre chargées des questions de droit et de justice, ainsi qu'au ministère après la Libération, renouait avec un usage contestataire du droit, mettant ses interprétations au service de la sauvegarde des libertés républicaines (27). Paul Coste-Floret rappelle en effet que l'Assemblée nationale a été dissoute le 9 octobre 1962 et que la loi de 1955 prévoyait la levée de l'état d'urgence dans un tel cas. La controverse qu'il ouvre se limite alors à des échanges verbaux avec le ministre de la Justice, Jean Foyer, et à la publication d'articles contradictoires dans Le Monde (28). Historiquement, l'état d'urgence a été levé le $1^{\text {er juin }} 1963$ à minuit, le général de Gaulle ayant lui-même affirmé que "nous en avons terminé avec la subversion" (29).

Néanmoins la thèse de Paul Coste-Floret a été retenue par le Conseil d'État en 1969. Examinant une requête portant sur la saisie d'un journal le 22 décembre 1962 , les Conseillers l'ont jugée illégale, car postérieure à la dissolution de l'Assemblée nationale dont ils estiment qu'elle a entraîné, effectivement, la fin de l'état d'urgence (30). Dans l'histoire tendue des rapports entre le général de Gaulle et le Conseil d'État, cette décision désavoue le gouvernement a posteriori mais, trop tardive pour porter à conséquence, elle ne contredit pas la prudence qui caractérisa

(26) A. Heymann-DoAt, Les libertés publiques et la guerre d'Algérie, Paris, LGDJ, 1972.

(27) L. ISRAËL, Robes noires, années sombres. Avocats et magistrats en résistance pendant la Seconde Guerre mondiale, Paris, Fayard, 2005.

(28) Juridiquement, le débat porte sur la relation entre l'ordonnance du 13 juillet 1962 prorogeant l'état d'urgence, qui a reçu a posteriori force de loi, et la loi précédente du 3 avril 1955. Pour Paul Coste-Floret, les dispositions de l'ordonnance, devenue loi, n'effacent pas celles de 1955, au contraire de ce que lui répondent ses détracteurs. Cf. les débats à l'Assemblée nationale, le 22 janvier 1963, ainsi que l'article de Paul Coste-Floret, "Feu l'état d'urgence ", dans Le Monde du 30 janvier 1963 et la réponse anonyme publiée le lendemain.

(29) Le Monde le rappelle opportunément, le 10 mai 1963, pour avertir le gouvernement que l'état d'urgence ne doit pas être prolongé une nouvelle fois.

(30) Arrêt " ministère de l'Intérieur contre les Éditions parisiennes associées et le sieur Devay ", 22 juin 1969, dossier d'archives au Centre des archives contemporaines de Fontainebleau, 19810257/17 (sous dérogation). 
les Conseillers en matière de contrôle des pouvoirs exorbitants accordés à l'Exécutif dans le contexte de la guerre d'Algérie, hormis l'arrêt Canal qui fit scandale (31). Cet arrêt témoigne néanmoins de l'acuité de la question de la levée d'un état d'exception, tant sur le plan juridique que sur le plan politique, et elle servit de leçon en 2005.

Au terme de ce premier parcours, l'état d'urgence prend un double visage. Il se présente comme un outil de répression politique, que ce soit contre les indépendantistes algériens en 1955 ou contre les partisans de l'Algérie française plongeant dans la haine pour le régime et le chef de l'État, après 1958. Mais il se présente aussi - et ce n'est là que le revers de la médaille - comme un outil de sauvegarde de la République. A condition, néanmoins, d'accepter le paradoxe voulant qu'il faudrait suspendre les libertés pour mieux les défendre contre leurs ennemis.

Ce paradoxe explique l'enjeu de la question de la levée de l'état d'urgence : une fois ce dernier déclaré dans des circonstances qui le légitimeraient, y compris pour des défenseurs de la République et des libertés individuelles ou collectives, comment apprécier le moment où cesse une telle justification? A partir de quand l'état d'urgence, présenté comme l'ultime recours du régime républicain en péril, devient-il, au contraire, lui-même, un danger? C'est le noud de toute controverse pour ceux qui ne le considèrent pas, d'emblée, par nature, comme liberticide.

La période ouverte par la tentative de putsch, en effet, montre qu'une fois introduit dans le droit français, l'état d'urgence est tombé dans l'escarcelle des moyens mis à la disposition des gouvernements, tentés d'en user comme n'importe quel autre, comme si le recours à l'état d'urgence s'était banalisé à force de prolongations. Et c'est bien parce qu'il est resté dans le droit comme tel qu'il a pu être réactivé en 2005.

\section{5, une loi pour l'opinion?}

Annoncer le recours à l'état d'urgence comme une remise en vigueur de "la loi de 1955 " efface la période gaulliste, pourtant lourde de modifications dans la rédaction ainsi que dans l'usage du texte, et établit un curieux raccourci à cinquante ans de distance. Les médias se sont montrés bien plus sensibles à la création de la loi dans le contexte des premiers mois de la guerre d'indépendance algérienne qu'à son usage dans les années 1958-1963, et ils ont surtout rappelé sa dernière application, en Nouvelle-Calédonie, en 1985, sur laquelle la documentation et les connaissances sont très pauvres (32).

(31) J. MASSOT, "Le rôle du Conseil d'État ", in J.-P. RIoux (dir.), La guerre d'Algérie et les Français, Paris, Fayard, 1990, p. 269-275. M.O. BARUCH et V. DuClERT (dir.), Justice, politique et République, de l'affaire Dreyfus à la guerre d'Algérie, Bruxelles, Complexe, 2002, p. 17 et suiv. S. THÉnAulT, "La guerre d'Algérie au Conseil d'État ", in Le Conseil d'État et l'outre-mer, à paraitre chez Dalloz.

(32) Pour le contexte général, A. Raluy, La Nouvelle-Calédonie, Paris, Karthala, 1990 ; J.-M. Regnault (dir.), François Mitterrand et les territoires français du Pacifique (1981-1988), Paris, Les Indes savantes, 2003. 
Dans les années 1980, le contexte calédonien a rejoué la conjoncture d'insurrection anti-coloniale algérienne. Le Front de libération nationale kanak et socialiste (FLNKS), qui a vainement placé ses espoirs en la victoire de François Mitterrand à l'élection présidentielle en 1981, a en effet connu une évolution menant son aile radicale, sous la conduite d'Eloi Machoro, à prendre les armes à l'automne 1984. Cependant, dans une logique de substitution à des pouvoirs publics jugés défaillants, les colons se sont organisés, se rendant même responsables de plusieurs tueries, dont celle de dix indépendantistes à Hienghène en décembre. Tandis qu'ils rejetaient le plan proposé par le haut commissaire de la République, Edgar Pisani, visant à organiser un référendum d'autodétermination, le GIGN assassinait Eloi Machoro, le 12 janvier 1985. C'est dans ces conditions que le gouvernement de Laurent Fabius a d'abord prévu, dans la loi du 6 septembre 1984 portant sur le statut de l'île, que l'état d'urgence pourrait y être instauré par le haut commissaire de la République, avant qu'il n'y soit effectivement déclaré au lendemain de la mort d'Eloi Machoro,

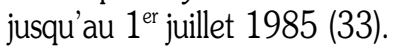

Il est notable, ici, que c'est un gouvernement socialiste qui recourt à l'état d'urgence, un gouvernement conforté par ses députés : 283 d'entre eux, sur 288, ont approuvé la déclaration et la prolongation de l'état d'urgence en Nouvelle-Calédonie, tandis que les communistes et les députés RPR la rejetaient en bloc (34). Les préventions des socialistes à l'égard de cet état d'exception se sont levées, depuis 1955, au point qu'en 2005, le PS s'est montré hésitant sur l'attitude à adopter. Les tenants d'une ligne sécuritaire, menés par le député de l'Essonne et maire d'Evry Manuel Valls, ont approuvé la décision de Dominique de Villepin.

L'attention particulière portée, aujourd'hui, à cette résurgence calédonienne de l'état d'urgence, en territoire et en contexte colonial, s'inscrit dans une conjoncture de polémiques aiguisées autour de ce pan de l'histoire de France. À la naissance du mouvement des "Indigènes de la République ", faisant du " continuum colonial " en France "une clé d'analyse très large " (35), a succédé le vote de la loi du 23 février 2005 , dont l'article 4 a suscité une vaste et profonde polémique, en particulier chez les historiens (36). La fracture coloniale, aussi, connaissait un franc succès en pointant l'absence de post-colonial studies en France, au contraire des pays anglosaxons qui seraient plus sensibles au sort de leurs minorités (37). Ce contexte de réminiscences fiévreuses du passé colonial français explique les réticences à appeler l'état d'urgence par son nom, au moment de sa proclamation. Il a été décrété en conseil des ministres le mardi 8 novembre 2005, au lendemain de son annonce par

(33) Loi no 84-821 du 6 septembre 1984, décret n 85-46 du 14 janvier 1985 et prolongation de l'état d'urgence par l'Assemblée nationale le 25 janvier 1985.

(34) 283 députés socialistes sur 288 , rejoints par cinq non-inscrits, ont voté pour, tandis que les 88 députés RPR et les 44 députés communistes votaient contre. A l'UDF, 55 députés sur 59 n'ont pas pris part au vote, les autres ont voté contre.

(35) J. RoBINE, "Les "Indigènes de la République" : nation et question post-coloniale. Territoires des enfants de l'immigration et rivalité de pouvoir ", Hérodote, $1^{\mathrm{er}}$ semestre 2006, p. 127.

(36) R. BERTRAND, Mémoires d'empire. La controverse autour du "fait colonial ", Broissieux, Éditions du Croquant, 2006.

(37) P. Blanchard, N. Bancel et S. Lemaire (dir.), La fracture coloniale, Paris, La Découverte, 2005. 
le Premier ministre, avant d'être, suivant l'ordonnance du 15 avril 1960, confirmé par l'Assemblée nationale jusqu'au 21 février 2006 (38).

Appliquer une loi créé pour s'opposer au déclenchement, par les Algériens, de leur " guerre de libération " et qui, pour la dernière fois, a été utilisée en NouvelleCalédonie dans un contexte de soulèvement indépendantiste, alors que le débat sur le passé colonial français et son héritage au présent occupe l'espace public, n'était-il pas maladroit ? D'autant que la nécessité de recourir à l'état d'urgence a été discutée : résultat d'une "erreur manifeste d'appréciation ", pour certains juristes, car la République n'était pas en péril, il aurait été, pour d'autres, seulement "disproportionné " (39). Au-delà de ces nuances, un consensus s'est dessiné pour demander, comme en 1955, "si l'on ne braque pas un canon pour écraser une mouche" (40). Un " proche " du ministre de l'Intérieur ne disait pas autre chose lorsqu'il laissait filtrer à la presse, dans un langage plus actuel : "C'est un truc à perdre la face " (41). Car, effectivement, l'état d'urgence a servi, principalement, à prononcer des couvre-feux dans les agglomérations touchées, alors que les maires des communes possèdent déjà ce pouvoir. Des rassemblements ont aussi été interdits, à Paris et à Lyon.

Il n'est pas évident que cet éventail de mesures nécessitait la déclaration de l'état d'urgence. Celle-ci, dès lors, relèverait-elle d'une stratégie politique, visant à satisfaire un électorat soucieux d'ordre et de sécurité ? Sur le moment, en tout cas, elle a permis au Premier ministre de se maintenir dans la compétition avec son ministre de l'Intérieur, Nicolas Sarkozy, pour l'élection présidentielle de 2007, en contribuant à lui forger une image d'homme capable de prendre ses responsabilités dans des circonstances difficiles. Il affirmait lui-même, dans sa déclaration télévisée, qu'il cherchait à créer un "choc ", propre à calmer les émeutiers et rassurer la majorité de l'opinion. $73 \%$ des Français, d'ailleurs, auraient approuvé la proclamation de l'état d'urgence (42).

Au contraire d'une maladresse, alors, le choix de prononcer l'état d'urgence reposait-il sur un calcul ? Si tel était le cas, la loi de 1955 n'aurait pas été choisie en dépit de son origine coloniale, malgré les blessures symboliques qu'elle était susceptible d'infliger à tous ceux qui se reconnaissent - à tort ou à raison, ce n'est pas le problème ici - comme les héritiers des victimes de la colonisation. Elle aurait été choisie, précisément, en raison de cette origine, pour adresser aux Français qui considèrent la lutte contre l'insécurité comme l'un des chantiers majeurs du présent un message de fermeté. Ainsi, pesant le pour et le contre, le Premier ministre pouvait estimer qu'il tirerait bénéfice de la proclamation de l'état d'urgence auprès de cette opinion majoritaire.

(38) Décrets nº 1386 et 1387 du 8 novembre 2005, déclarant l'état d'urgence et définissant les zones où il s'applique. Ils ont été confirmés à l'Assemblée par 346 voix contre 148, grâce aux voix de l'UMP et de l'UDF, tandis que les élus de l'opposition de gauche, socialistes, membres des Verts et communistes, ont voté contre.

(39) "Inutile? Nécessaire? L'avis de quatre professeurs de droit ", Le Monde, 16 novembre 2005.

(40) A. CHENEBOIT, "Tribune", art. cit.

(41) Citation anonyme dans Le Monde du 9 novembre 2005.

(42) D'après un sondage de l'Institut CSA. 
Il est un sujet, toutefois, sur lequel le présent ne rejoua pas le passé : celui de la levée de l'état d'urgence. Dès le 10 novembre 2005, la presse signalait que "l'Elysée et Matignon réfléchissent déjà à la meilleure manière de sortir de l'état d'urgence" (43). Si sa proclamation était considérée comme payante auprès de l'opinion, une application durable dans le temps risquait d'être contre-productive. Des juristes, par ailleurs, saisirent immédiatement le Conseil d'État, qui se prononça ainsi pendant la période d'application de l'état d'urgence et non des années plus tard. La rapidité de cette requête a permis aux Conseillers d'anticiper le moment où l'état d'urgence devrait cesser et leur arrêt a fait planer la menace d'une censure juridique, au cas où le pouvoir politique se prendrait à renouveler un état d'urgence devenu illégitime.

C'est Frédéric Rolin, alors professeur de droit public à l'Université d'Eury, qui, le premier, a contesté la proclamation de l'état d'urgence devant le Conseil d'État, au motif qu'elle était "exagérée " au regard des événements en cours. Le 14 novembre 2005, les Conseillers l'ont débouté, en estimant au contraire que "l'aggravation continue depuis le 27 octobre 2005 des violences urbaines, leur propagation sur une partie importante du territoire et la gravité des atteintes à l'ordre public " (44) le justifiaient entièrement. Ce faisant, ils fondaient logiquement la légitimité du régime d'état d'urgence sur les circonstances au nom desquelles il a été instauré. Ils ouvraient donc la possibilité de requêtes ultérieures le contestant, au nom d'une modification de ces circonstances, leur donnant l'occasion d'apprécier, de nouveau, son opportunité (45).

Des juristes ne pouvaient pas s'y tromper : la seconde requête a été déposée par un collectif de soixante-quatorze d'entre eux, représentés par Frédéric Rolin. Examinée le 9 décembre 2005, cette requête arguait de la fin des violences pour réclamer la suspension de l'état d'urgence. Entérinant son raisonnement précédent et renforçant son message à l'attention d'un pouvoir politique ainsi prévenu, le Conseil a concédé que "les circonstances qui ont justifié la déclaration de l'état d'urgence avaient sensiblement évolué " (46). Néanmoins il a rejeté la requête en s'appuyant sur le risque d'un nouvel embrasement à l'occasion des fêtes de fin d'année. Le message était clair : si celles-ci se passaient dans le calme, le gouvernement n'aurait plus aucune raison de maintenir l'état d'urgence en vigueur et une nouvelle requête aurait toutes les chances d'aboutir. C'est dans ces conditions que l'état d'urgence a été levé, le mercredi 4 janvier 2006. La France sortait de l'exception avant la date prévue par le vote de l'Assemblée nationale.

Au terme de ces cinquante ans, la loi d'état d'urgence se présente-t-elle comme une loi coloniale, dont la réactivation s'inscrirait dans une logique post-coloniale,

(43) D'après Le Monde du 10 novembre 2005.

(44) Le Monde du 15 novembre 2005.

(45) Sur l'art des Conseillers de jouer du droit : B. LaTour, La fabrique du droit. Une ethnographie du Conseil d'État, Paris, La Découverte, $2^{e}$ éd., 2004.

(46) D'après Le Monde du 9 décembre 2005. 
visant à soumettre les immigrés et leurs familles, aujourd'hui, à un traitement analogue à celui réservé autrefois aux Algériens? C'est dans cette perspective qu'a été dénoncé, entre autres, le recours à l'état d'urgence en 2005, en raison d'un contexte favorable à la promotion de cette grille de lecture.

Il semble néanmoins que l'interprétation du recours à l'état d'urgence comme une manifestation de post-colonialisme ne suffit pas à l'analyser. Comment intégrer, dans ce cas, la période 1958-1963, où la loi de 1955 fut modifiée et longuement appliquée, contre les factieux sortis des rangs des partisans de l'Algérie française? L'état d'urgence est aussi - et au moins autant - une loi de répression politique qu'une loi coloniale. Les deux, d'ailleurs, ne sont pas contradictoires. La mise au point de cet état d'exception puise ainsi aux sources du régime républicain français, né dans les circonstances de la Révolution et en particulier celles de la Terreur déclenchée contre ses ennemis. L'exposé des motifs recourt à une notion fondamentale, datant de cette période : celle de "hors la loi ". Or ce dernier n'est pas seulement celui qui a enfreint la légalité, comme le sens commun l'entend généralement. Il est, à l'origine, celui qui, contestant l'ordre de la République, se serait placé de lui-même en dehors de sa Loi. L'idée est que, s'il ne reconnaît pas ce régime, il ne peut plus prétendre aux garanties et protections qu'offre le droit commun. Hors de la Loi commune, il peut légitimement être soumis à une législation d'exception. La procédure de "mise hors de la loi" a ainsi été créée par un décret du 19 mars 1793 contre les insurgés vendéens favorables au retour de la monarchie. Suivant cette procédure, ils étaient passibles, sur "une simple déposition orale ou écrite de deux témoins", d'un "tribunal jugeant "révolutionnairement" (sans jurés) pour décider de l'application de "la seule peine possible, la mort " (47).

Par la notion de "hors la loi ", cependant, l'état d'urgence ramène aussi, au présent, au contexte international. Il suffit, en effet, de substituer à cette notion désuète celle de "terroriste" pour dérouler le fil d'un raisonnement analogue. De nos jours, l'état d'urgence, en tant qu'état d'exception destiné à combattre ceux qui rejettent l'ordre politique, se trouve relégitimé par les discours et les dispositions anti-terroristes qui ont fleuri, depuis le 11 septembre 2001, outre-Atlantique, et plus récemment outre-Manche, quand ce n'est pas en France même. Il répond à une demande de sécurité généralisée dans ce contexte, même si son application, fin 2005 , ne doit rien à une quelconque action terroriste.

L'état d'urgence s'enracine ainsi dans l'histoire de la répression en France, visant des mouvements politiques et/ou terroristes, recourant au droit et à la justice, au nom de la défense de la République contre ceux qui voudraient la mettre en péril. Aux colonies, certes, mais pas seulement : anarchistes et communistes y firent face aussi. Dans tous les cas et suivant des modalités variables sous la III ${ }^{e}$, la IVe et la $V^{e}$ République, il s'agit, pour l'autorité politique, de recourir exceptionnellement à

(47) J.-C. FARCY, L'histoire de la justice française de la Révolution à nos jours, Paris, PUF, 2001, p. 377. Il renvoie à la thèse de droit d'E. de MARI, La mise hors la loi sous la Révolution française (19 mars 1793-9 thermidor an II), Université Montpellier I, 1991. 
une répression légale pour tenter de "juguler une crise, de maintenir le régime et d'empêcher un changement perçu comme séditieux, factieux ou subversif " (48).

La proclamation de l'état d'urgence donne-t-elle un sens, alors, aux violences urbaines de novembre 2005, que leurs acteurs ont laissées sans signification politique ? Emeutes et désordre à réprimer, certes, mais avec quelle portée ? Une portée subversive, semble dire le recours au texte, conçu pour ceux qui se sont situés en dehors du contrat social, en dehors de la République et en dehors de la nation. Effectivement, si ces événements ont des causes socio-économiques, s'ils résultent de tensions exacerbées par des discriminations en partie liées à l'héritage du passé colonial français, c'est bien une crise de la nation au sens d'une "représentation géopolitique chargée de valeurs et d'histoire que partagent, au-delà de leurs convictions politiques contradictoires et de leurs inégalités sociales, un grand nombre d'hommes et de femmes" (49) qu'ils ont exprimée.

(48) F. MONIER, "Le régime intangible? République et conspirations", Politix, troisième trimestre 1999, p. 25.

(49) Y. LACOSTE, Vive la nation, Paris, Fayard, 1997, cité par B. GIBLIN, «Fracture sociale ou fracture nationale ? De la gravité des violences urbaines de l'automne 2005 ", Hérodote, $1^{\text {er }}$ trimestre 2006, p. 87. 